\title{
Zur Biologie des Potamoplanktons auf Java.
}

\author{
Von \\ Dr. Paul van Oye (Tasikmalaja - Java).
}

Über das Potamoplankton der Tropen ist bis jetzt so gut wie nichts erschienen, und das wenige, was man in den verschiedenen Publikationen hierüber zerstreut finden kann, ist meistens nur faunistischer und floristischer Art. Bis jetzt ist kein einziger Untersucher der Biologie des tropischen Potamoplanktons längere Zeit nachgegangen.

E. Lemmermann ${ }^{1}$ ) hat das Potamoplankton des Menam-Flusses in Siam untersucht, und zwar das Material, welches Prof. Dr. H. Schauinsland während seiner Reise 1906 gesammelt hatte. Diese Arbeit ist natürlich eine rein systematische, und die Ergebnisse, welche Lemmermann am Ende seiner Publikation gibt, haben dann auch keinen biologischen Wert; um so mehr als Lemmermann nur angibt, daß die Reise 1906 gemacht und das Material bei Pakman gefischt wurde, jedoch weder Tag noch Monat erwähnt, wann die Planktonproben entnommen wurden. Hierdurch wird jeder Vergleich unmöglich.

Nur Fritsch ${ }^{2}$ ) hat einiges über die Ökologie der Süßwasseralgen von Ceylon beobachtet, was für den Begriff der allgemeinen Biologie des Süßwasserplanktons der Tropen sehr wertvoll ist. Aber der Aufenthalt von Fritsch auf Ceylon war auch für diese Frage, ebenso wie für das Studium der Okologie des Planktons der Reisfelder ${ }^{3}$ ) von zu kurzer Dauer, um uns einen Einblick in die Biologie und die Ökologie des Planktons geben zu können.

${ }^{1}$ ) Lemmermann, E., Das Phytoplankton des Menam. Hedwigia XLVIII. 1909, s. 126 .

$\left.{ }^{2}\right)$ Fritsch, F. E., A General Consideration of the Subaërial and Fresh-water Algal Flora of Ceylon. A Contribution to the Study of Tropical Algal Ecology. Proc. roy. Soc. London, Vol. LXXIX, 1907, \$. 197.

3) van Oye, P., Iets over de microfauna en -flora der rijstvelden in verband met de praktijk. Natuurwetenschappelijk Tijdschrift, Antwerpen 1921, S. 121. 
Fritsch sagt hierüber selbst (S. 199): "Since my stay in the island was of brief duration (August 21 to November 10, 1903) my account of the algal vegetation is necessarily very incomplete in places. I rarely stayed more than two or three days in any one locality, so that although able to familiarise myself with a wide range of climatic conditions and their influence on algal growth, I was unable to enter into detailed study of any given problem. Moreover, my observations, of course, merely deal with one phase of the algal vegetation, and it is possible that many other peculiarities would come to light if the period of observation could be extended over an entire year. I am however inclined to think that algal growth in the tropics will not show so marked a periodicity as obtains in our parts."

Nun ist der letzte Satz schon ganz unrichtig, wie ich in den bier folgenden Zeilen für das Potamoplankton zeigen kann und wie ich auch für das Plankton der Sitoes, der größeren Wasseransammlungen, in' einer demnächst erscheinenden Arbeit zeigen werde.

Um jedoch jeder Verwirrung vorzubeugen, muß ich von Anfang an darauf hinweisen, daß auf Java jedes Wasser ein eigenes Plankton besitzt, insofern als das Verhältnis der Planktonarten meistens ein ganz anderes ist. Es ist, wie Fritsch vermutete: "They othe aquatic formations) will probably in many cases be distinguished by the way in which the different members of the formation grow together, rather than by any individual species."

Dies hat sich nun so richtig erwiesen, daß der folgende Satz von Fritsch bis jetzt für Java - und ich bin seit mehr als drei Jahren in der Lage, das Süßwasserplankton verschiedenartiger Gewässer zu untersuchen - sich als ungültig erwiesen hat, nämlich wo dieser Autor sagt: "It even seems quite possible to imagine two algal formations of almost identical, specific composition, but differing from one another biologically or physiologically."

Wie aus folgenden Zeilen hervorgeht, und auch aus meinen anderen Arbeiten über die Biologie und Ökologie der Mikroorganismen in allgemeinen auf Java, ist dies niemals der Fall.

In meiner kurzen Notiz über das Potamoplankton des TjiliwoengFlusses bei Batavia (Niederl.-Ostindien) ${ }^{1}$ ) habe ich darauf hingewiesen, wie abweichend sich das Plankton im Tjiliwoeng-Flusse bei Batavia

') van Oye, P., Über das Plankton des Tjiliwoeng-Flusses bei Batavia (Niederl.Ostindien). Intern. Rev. gesamt. Hydrob. und Hydrogr., Bd. IX, 1920, S. 160-165. 
von allem verhält, was wir über das Potamoplankton der gemäßigten Zonen wissen. Damals habe ich noch viele Fragen offenlassen müssen.

Da ich nun neben gelegentlichen Untersuchungen, längere Zeit nacheinander Gelegenheit hatte, das Plankton von drei ökologisch sehr verschiedenen strömenden Gewässern Javas zu untersuchen, glaube ich jetzt in der Lage zu sein, einige der damals gestellten Fragen erörtern zu können.

Obwohl ich schun mebrmals auf einige Punkte hingewiesen habe in Verbindung mit den genauen Angabèn von Ort und Zeit der gefundenen Mikroorganismen, will ich hier nochmals auf dieselben zurückkommen.

Die meisten Autoren geben in dieser Hinsicht einfach die Fundstellen an, andere finden es selbst schon genügend, zu erwähnen, daß bestimmte Mikroorganismen in den Tropen gefunden worden sind. So sagt Schmidle ${ }^{1}$ ) in seiner Arbeit über die Süßwasseralgen von Ostindien, nachdem er erwähnt hat, daß das bearbeitete Material durch Herrn Professor Dr. A. Hansgirg in Vorderindien und Ceylon im Jahre 1895 gesammelt wurde, über die Standorte: „Denn es ist ja für die Wissenschaft völlig gleichgültig, die genauen zufälligen Standorte solcher Ubiquisten zu kennen." Dies ist insofern unrichtig, als es sich nicht nur um einen zufülligen und gleichgültigen genauen Standort handelt, sondern auch um eine genaue Zeitangabe. Durch seine verkehrte Auffassung in dieser Hinsicht hat die durch Schmidle weiter aufgegebene Liste von ,ubiquitäre oder in den Tropen verbreiteten Algen" nicht den mindesten biologischen Wert.

Auch Fritsch ${ }^{2}$ ) ist nach seinen Untersuchungen auf Ceylon ganz und gar derselben Ansicht und gibt selbst, wie ich erst nach Abfassung der Arbeit gesehen habe, auch dieselben Worte von Schmidle als Beispiel an.

So ist es auch, wie schon erwähnt, mit den Untersuchungen von Lemmermann ${ }^{3}$ ) über das Phytoplankton des Menams, und es wäre nicht schwierig, diese Liste noch zu verlängern.

Alle diese Arbeiten sind zu sehr vom rein systematischen Standpunkt aus gesehen. Wer in den Tropen selbst gearbeitet hat, weiß, daß systematische Arbeiten hier, wenn nicht unmöglich, so doch äußerst schwierig

') Schmidle, W., Über einige von Professor Hansgirg in Ostindien gesammelte süßwasseralgen. Hedwigia, Bd. XXXIX, 1900, S. 160.

2) Fritsch, F. E., The Subaerial and Freshwater Algal Flora of the Tropies. Ann. of Bot., Vol. XXI, 1907, S. $23 \overline{5}$.

3) Lemmermann, E., I. c. Hedwigia XLVIII, 1909. 
sind wegen des Mangels an Literatur, Vergleichungsmaterial und Hilfe in jeder Beziehung.

Erinnern wir an die systematischen Arbeiten von Ch. Bernard ${ }^{1}$, die leider durch $W_{e s t^{2}}$ ) zu ungerecht beurteilt wurden. Obwobl wir die Schreibweise von West. stark verurteilen müssen (West scheint keine Ahnung davon zu haben, welche Schwierigkeiten planktologische Untersuchungen in den Tropen bieten), bleiben seine Bemerkungen jedoch richtig; es ist hiermit der Beweis geliefert, daß eine Systematik der niederen Organismen in den Tropen zur Zeit noch höchst selten möglich ist; aber dagegen beweisen auch die Unrichtigkeiten in der Arbeit von Fritsch, wovon ich schon einzelne erwähnt babe, daß die biologischen und ökologischen Untersuchungen nur an Ort und Stelle und bei einem längeren Aufentbalt möglich sind.

Dies war für mich die Veranlassung, meine Arbeit anders aufzufassen. Ich habe stets nur rein biologische und ökologische Fragen vor Augen gehabt, und was sich hierbei an faunistischen und floristischen Tatsachen herausstellte, wurde einfach notiert. Diese rein systematischen Ergebnisse werden an anderer Stelle als Beiträge zur Kenntnis der Mikrıfauna und -flora von Java mit genauer Angabe ron Zeit und Ort der Fänge mitgeteilt werden.

Wer die Arbeiten von Jadwiga Woloszynskas) und R. Gutwin$\mathrm{ski}^{4}$ ) einsieht, wird sofort erstaunt sein über die Fülle neuer Arten und Varietäten, welche diese Autoren in dem wenigen Material gefunden haben. Da ich nun seit mehr als drei Jahren in Java aus Seen, Teichen, Strömen und Binnengewässern aller Art Plankton untersuche, ist es selbstverständlich, daß viele Arten vorgekommen sind, die ich als neu

1) Beraard, Ch., Protococcacées et Desmidiées d'eau douce récoltées à Java. Batavia 1908.

id. Sur quelques Algues unicellulaires d'Eau douce récoltées dans le Domaine Malais. Buitenzorg 1909.

2) West, G. S., Botanical Synonyms in the Desmidiaceae and Protococcoldeac. Journ. of Botan., Vol. XLVII, 1909, S. 60.

3) Woloszynska, Jadwiga, Das Phytoplankton einiger javanischer Seen mit Berücksichtigung des Sawa-Planktonø. Bull. Intern. Acad. se. Cracovie, Ser. B, 1912, s. 649.

4) Gutwinski, R., Addimenta ad Floram Algarum Indiae Batavorum cognoscendam. Dissert. mathem. et physic. Acad. Cracovensis, Tomo XXXIX, 1901, S. 287.

id., De Algis a Dr. M. Raciborski anno 1889 in Insula Java collectis. Bull. Int. Acad. sc. Cracovie, 1902, S. 575. 
angeseben habe, aber die Beschreibung dieser vermutlich neuen Arten habe ich unterlassen; womöglich wird dies unter besseren Verhältnissen später stattfinden. Die biologischen Tatsachen stätzen sich allerdings doch meistens auf große Gruppen, und um die biologischen Tatsachen ist es mir vorläufig nur zu tun. In dieser Hinsicht muß ich sagen wie Fritsch: "Specific determinations would in most cases be quite valueless from the present point of view, and would moreover, have very inconsiderably delayed publication".

Aber es ist Tatsache, daf, um die biologischen Schlüsse aus floristischen und faunistischen Listen ziehen zu können, man genaue Angaben von Platz, Tag, Monat, eventuell Jabreszahl, Höhe, meteorologische Umstände usw. nötig hat und in dieser Hinsicht sind fast alle Publikationen ron anderen Antoren mangelhaft.

Um den Wert für die Biologie, selbst bei unrichtigen Bestimmungen, deutlich anzuzeigen, will ich noch auf die Arbeit von M. Treub über die Wiederbesiedlung von Krakatau hinweisen. Hier sind fast alle Bestimmungen fon Myxophyceen den Arten nach unrichtig gewesen, aber seine biologischen Beobachtungen müssen jedoch als richtig anerkannt werden.

Endlich muf ich noch in bezug auf meine eigenen Artbestimmungen erwähnen, daß alle meine Listen nur diese Arten angeben, welche ich mit der größtmöglichen Sicherheit, ich wage selbst zu sagen, mit absoluter Sicherheit, mit Hilfe der mir zur Verfügung stehenden Mittel machen konnte. Meine Listen geben also kein genaues faunistisches oder floristisches Bild, sondern nur ein rein biologisches, wobei zu erwähnen ist, daß die genannten Aıten mit möglichster Sicherheit bestimmt wurden und die zweifelhaften Arten nicht aufgenommen sind. Wo dies für die biologische Beurteilung nötig war, habe ich hier und da ganze Gruppen mit einem Namen erwähnt, so z. B.: Copepoden, viel, Dies ist stets der Fall gewesen, wenn die genannte Gruppe ron einem biologischen Standpunkt von besonderem Interesse war und ich nicht in der Lage gewesen bin, die Arten selbst sicher bestimmen zu können.

Wo J. Comère' 1906 für Frankreich noch mit Recht sagen konnte: „Des observations longues et nombreuses pourront seules conduire à la formation d'une sorte de calendrier phyculogique" ist es nicht nötig.

\footnotetext{
1) Comère, J., Obscrvations sur la pérjodicité du développement de la flore algologique dans la région toulousaine. Bull. soc. bot. de France, T. LIII, 1906, s. 390 .
} 
nochmals darauf hinzuweisen, daß unsere Kenntnisse von der Periodizität des Planktons in den Tropen noch ganz unvollständig sind und sich erst in einem Anfangsstadium befinden.

Die strömenden Gewässer Javas habe ich in zwei Kategorien eingeteilt ${ }^{1}$ ): Die Bergströme und die Flachlandströme. Zur Zeit muß ich jedoch die letzten wieder in zwei Gruppen einteilen, nämlich in tiefe und untiefe Flachlandströme.

Obwohl die Tiefe der Flachlandströme nicht das charakteristischste Morkmal ist, muß ich sie vorläufig noch zur Kennzeichnung gebrauchen; denn bis jetzt erscheint mir die Tiefe noch als das bequemste und einfachste Merkmal zur Unterscheidung der zwei Gruppen.

Ist die Einteilung der fließenden Gewässer Javas eine morphologische und topographische, so ist sie zugleich auch eine biologische, und dies will ich hier näher untersuchen und erörtern. Dabei stellt sich auch heraus, daß das Plankton der strömenden Gewässer einer bestimmten Periodizität unterworfen ist und sie auch auf Grund ihres Phytoplanktons eingeteilt werden können.

Gehen wir aber erst den Resultaten der Untersuchung nach.

Beobachtet und untersucht wurden: der Bergstrom Tjikoenir bei Tawangbanteng, ungefähr $10 \mathrm{~km}$ ron Tasikmalaja. Ein Bach, ebenfalls bei Tawangbanteng und unweit vom Flusse Tjikoenir, ja selbst in engster Beziehung mit diesem, und endlich ein untiefer Flachlandstrom, Tjimoeloe, bei Tasikmalaja selbst.

Der Tjikoenir-Fluß ist ein echter tropischer Bergstrom. Wie alle Ströme auf Java ist er von bedeutender Größe in der Regenzeit, doch fast wie ein Bach in der Trockenzeit. Das Bett besteht aus Steinen und großen Lavablöcken, zwischen welchen das Wasser brausend vorwärtsströmt.

Jedesmal habe ich in der auf anderer Stelle von mir beschriebenen Weise $\left.{ }^{2}\right) 100 \mathrm{l}$ Wasser durch ein kleines Netz aus Müllergaze Nr. 25 filtriert und den Rückstand in einer Lösung von 10 Proz. Formalin aufbewahrt. Zu Hause wurde das Setzvolumen in gewöhnlichem Meßzylinder festgestellt und der Inhalt mikroskopisch untersucht.

Hierbei ist zu erwähnen, daß die Diagnosen einiger Arten wie Spi-

1) van Oye, P., Einteilung der Binnengewässer Javas. Intern. Rev. der Hydrob. und Hydrogr.. X, 19थ2, S. 7 .

2) van Oye, P., Einige sehr einfache Methoden für planktologische Untersuchungen in den Tropen. Mikrokosmos 1920-21, Heft 11. 
rogyra und Oedogonium nicht immer gestellt werden konnten, da nicht immer Exemplare mit den nötigen Geschlechtsmerkmalen vorhanden waren, weshalb die Benennung sehr oft unterbleiben mußte. Die weiter unten angeführten Listen geben, wie schon erwähnt, nur dasjenige an, was mit Sicherheit bestimmt werden konnte. Wie ich diese Listen betrachte und welchen Wert nach meinem Urteil diese Angaben haben. sollen, habe ich schon im Detail an anderer Stelle auseinandergesetzt ${ }^{1}$ ).

Für den Bergstrom Tjikoenir sind die Ergebnisse meiner Untersuchungen folgende:

16. Oktober 1920. Pro $100 \mathrm{l}$ Wasser $1 \mathrm{ccm}$ Satz.

Viel Schlick und wenig Detritus.

Draparnaldia plumosa (Vaucher) C. A. Agardha 2 .

Surirella splendida Kuetzing.

Bacillaria paradoxa (Gmelin) Gruenow.

Nitzschia sigmoidea (Nitzsch) W. Smith.

Ulothrix subtilis Kuetzing.

Spirogyra tenuissima (Kuetzing) Kirchner.

30. Oktober 1920. Pro $100 \mathrm{l}$ Wasser $1 \mathrm{ccm}$ Satz.

Viel Schlick und wenig Detritus.

Stigeoclonium tenue Kuetzing.

Spirogyra spec.

17. November 1920. Pro $100 \mathrm{l}$ Wasser $1 \frac{1}{2}$ ccm Satz.

Viel Schlick und wenig Detritus.

Polyarthra platyptera Ehrenberg.

Anurea aculeata Ehrenberg var: valga Ehrenberg.

Microspora stagnorum (Kuetzing) Lagerheim.

Draparnaldia plumosa (Vaucher) C. A. Agardh.

Spirogyra crassa Kuetzing.

Surirella splendida Kuetzing.

Copepoden.

8. Dezember 1920. Pro $100 \mathrm{l}$ Wasser $6 \mathrm{ccm}$ Satz.

Beinahe ausschließlich Schlick, sehr wenig Detritus.

Spirogyra crassa Kuetzing.

') van Oye, P., Een nieuwe richting bij de studie der lagere wicren. Teysmannia, 1919, S. 393.

2) Meine teilweise in Nachfolge amerikanischer Beispiele einigermaßen geänderte Schreibweise der wissenschaftlichen Nomenklatur werde ich an anderer Stelle näher besprechen. 
Surirella splendida Kuetzing.

Tabellaria floculosa (A. G. Roth) Kuetzing.

Merismopedium tenuissimum Lemmermann.

Stauroneïs gracilis W. Smith.

Stigeoclonium tenue Kuetzing.

7. Januar 1921. Pro 1001 Wasser $2 \mathrm{ccm}$ Satz.

Schlick und Detritus.

Arcella vulgaris Ehrenberg.

Surirella splendida Kuetzing.

Spirogyra crassa Kuetzing.

Arcella vulgaris Ehrenberg var. angulosa (Perty) Leidy.

Cladophora glomerata (Linnaeus) Kuetzing.

Cladophora fracta (Vahl) Kuetzing.

13. Januar 1921. Pro $100 \mathrm{I}$ Wasser $8 / 4 \mathrm{ccm}$ Satz.

Wenig Schlick und Detritus.

Surirella splendida Kuetzing.

Synedra ulna Ehrenberg.

Cladophora glomerata (Linnaeus) Kuetzing.

Oedogonium spec.

23. Januar 1921. Pro $100 \mathrm{~J}$ Wasser $1 / 2$ ccm Satz.

Wenig Schlick und Detritus.

Surirella splendida Kuetzing.

Melosira varians C. A. Agardb.

Synedra capitata Ehrenberg.

Synedra gracilis Kuetzing.

Oedogonium spec.

11. Februar 1921. Pro 1001 Wasser $3 / 4 \mathrm{ccm}$ Satz.

Viel Schlick und Detritus.

Surirella splendida Kuetzing.

Melosira varians C. A. Agardh.

Arcella vulgaris Ehrenberg.

Difflugia corona Wallich.

2. April 1921. Pro 1001 Wasser 1 ccm Satz.

Viel Schlick und Detritus.

Surirella splendida Kuetzing.

Arcella vulgaris Ehrenberg var. angulosa (Perty) Leidy. 
Synedra ulna (Nitzsch) Ehrenberg.

Cathypna Iuna Ehrenberg 1 Exemplar.

24. Mai 1921. Pro $100 \perp$ Wasser $1 / 4 \mathrm{ccm}$ Satz.

Sehr wenig Schlick und Detritus.

Cladophora glomerata (Linnaeus) Kuetzing.

Nitzschia sigmoïdea (Nitzsch) W. Smith.

Navicula affinis Ehrenberg var. amphirhynchus (Ehrenberg)

Gruenow.

9. Juni 1921. Pro $100 \mathrm{l}$ Wasser $1 \mathrm{ccm}$ Satz.

Sehr wenig Schlick und Detritus.

Draparnaldia plumosa (Vaucher) C. A. Agardh.

Surirella splendida Kuetzing.

Bacillaria paradoxa (Gmelin) Gruenow.

Hydrodiction utriculatum Roth.

Draparnaldia glomerata (Vaucher) C. A. Agardh.

Spirogyra crassa Kuetzing.

Synedra limnetica Lemmermann.

20. Juni 1921. Pro 1001 Wasser $1 / 2$ ccm Satz.

Fast kein Schlick und Detritus.

Draparnaldia plumosa (Vaucher) C. A. Agardb.

Spirogyra crassa Kuetzing:

Spirogyra weberi Petit.

Oedogonium spec.

Stigeoclonium tenue Kuetzing.

Hydrodictyon utriculatum Roth.

Viel Rhizopoden.

Arcella vulgaris Ehrenberg.

Difflugia lobostoma Leidy.

Nebela collaris Leidy.

Difflugia constricta (Ehrenberg) Leidy.

Cosmariun subcrenatum Hantzsch.

Synedra actinastroïdes Lemmermann.

Bacillaria paradoxa (Gmelin) Gruenow.

Arcella vulgaris Ehrenberg var. angulosa (Perty) Leidy.

Diese Listen lehren uns, daß im Bergstrom Tjikoenir bei Tawangbanteng von November 1920 bis Juli 1921 mit Sicherheit folgende Mikroorganismen vorkamen: 
Myxophyceae:

Merismopedium tenuissimum Lemmermann.

Diatomeae:

Nitzschia sigmoidea (Nitzsch) W. Smith.

Surirella splendida Kuetzing.

Bacillaria paradoxa (Gmelin) Gruenow.

Tabellaria floculosa (A. G. Rotb) Kuetzing.

Stauroneïs gracilis W. Smith.

Synedra ulna Ehrenberg.

" capitata Ehrenberg.

$" \quad$ actinastroïdes Lemmermann.

$" \quad$ gracilis Kuetzing.

" limnetica Lemmermann.

Navicula affinis Ehrenberg var. amphirhynchus (Ehrenberg)

Gruenow.

Melosira varians C. A. Agardh.

Desmidiaceae.

Cosmarium subcrenatum Hantzsch.

Oedogonium spec.

Chlorophyceae.

Draparnaldia plumosa (Vaucher) C. A. Agardh.

" glomerata (Vaucher) C. A. Agardh.

Cladophora glomerata (Linnaeus) Kuetzing. fracta (Vahl) Kuetzing.

stigeoclonium tenue Kuetzing.

Spirogyra crassa Kuetzing.

$" \quad$ weberi Petit.

$" \quad$ tenuissima Kuetzing.

Microspora stagnorum (Kuetzing) Lagerheim.

Ulothrix subtilis Kuetzing.

Hydrodictyon utriculatum Roth.

Rhizopoda.

Arcella rulgaris Ehrenberg.

Arcella vulgaris Ehrenberg var. angulosa (Perty). Leidy.

Difflugia corona Wallich.

" lobostoma Leidy.

" constricta (Ehrenberg) Leidy.

Nebela collaris Leidy. 
Rotatoria.

Cathypna luna Ehrenberg. Polyarthra platyptera Ehrenberg.

Anurea aculcata Ehrenberg var. valga Ehrenberg.

\section{Copepoda.}

Einige.

Die Copepoden konnten wegen Mangels an der nötigen Literatur nicht bestimmt werden.

Aus der Liste der gefundenen Arten ist jedoch zu ersehen, daß die Diatomeen und die Chlorophyceen die Überhand haben.

Bis jetzt wurden im Plankton der Tjikoenir 35 Mikroorganismen gefunden, und zwar:

\begin{tabular}{|c|c|c|c|}
\hline Myxophyceae & & & \\
\hline Diatomeae. & & & \\
\hline Desmidiaceae. & & & \\
\hline ChIorophyceae & & & \\
\hline Rhizopoda. & & & \\
\hline Rotatoria. . & & & \\
\hline
\end{tabular}

Weiter kann man in der Angabe der Ergebnisse sehen, daß die Chlorophrceen in den Monaten Oktober, November, Dezember und Januar viel vorkommen, doch geht aus den Planktonproben selbst hervor, daß diese Gruppe im Monat Januar quantitativ abnimmt. Dagegen verschwinden die Chlorophyceen ganz und gar im Monat Februar, um erst im Mai wieder zu erschtinen. Die hier angeführten Ergebnisse erlauben an sich selbst nicht die oben formulierten Schlüsse so positiv anszudrücken, aber auf Grund aller meiner Untersuchungen, woron verschiedene in Druck sind und andere demnächst erscheinen werden, glaube ich berechtigt zu sein, die Folgerung zu formulieren, wie ich es getan habe.

Weiter lehren uns die Ergebnisse, daß wir nit dem Anfang der Regenperiode, also etwa Anfang September, ein Aufblühen des Planktons in den Bergströmen sehen können; daß weiter das Plankton der Bergströme sich qualitativ ron allen anderen Bezirken unterscheidet dadurch, daß hier viel Chlorophyceen rorkommen, die sonst nirgendwo in dieser Anzahl zu finden sind, vor allem die Genera Draparnaldia, Stigeoclonium, Cladophora, Oedogonium, Ulothrix und Spirogyra. Als Ursachen müssen hier angesehen werden der große Sauerstoffgrehalt des brausenden Wassers und die große Helligkeit des Wassers 
der Bergströme, zwei ökologische Faktoren, die der Entwicklung dər rorkommenden Chlorophyceen sehr günstig sind.

Die Diatomeen kommen eigentlich während des ganzen Jahres ror, doch ist ihre Anzahl etwas größer in den Moiaten Dezember und Januar.

Die Rhizopoden erscheinen erst im Januar und kommen in den Monaten Oktober, November und Dezember gar nicht vor.

Quantitativ geben unsere Untersuchungen folgende Setzrolumina:

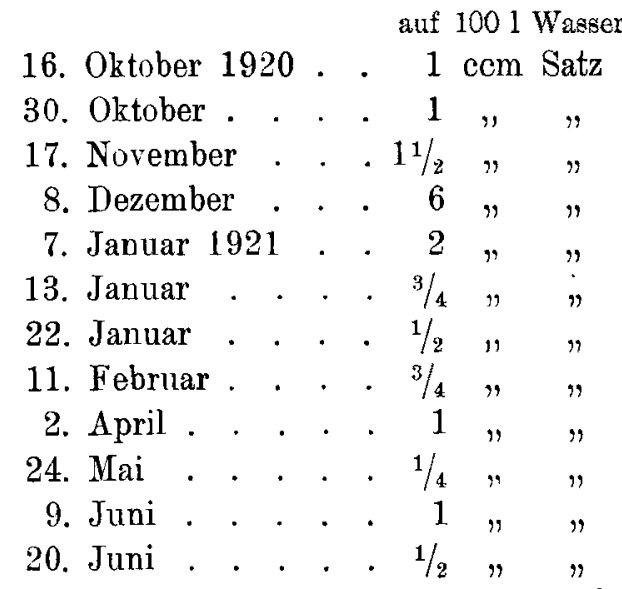

Diese Ziffern werden wir weiter unten zusammen mit den Ergebnissen der Tjimoeloe und des Baches besprechen.

Wenden wir uns jetzt zu den Untersuchungen der Tjimoeloe. Dies ist ein Flachlandstrom, und zwar nach meiner Einteilung ein untiefer Flachlandstrom.

Hiermit ist gesagt, daß das Wasser langsam fließt und vor allem nie braust, daß das Bett untief ist, so daß zu jeder Zeit ein Mensch stehend durch den Strom gehen kann, und endlich daß das Bett aus Sand, Erde, Laterit, sehr feinen Kieseln oder ähnlichen Bestandteilen besteht, doch nie aus größeren Steinblöcken.

Die Tjimoeloe läuft durch Tasikmalaja und führt allen Abfall von einem großen Teil dieses Ortes fort.

Ebenso wie dies der Fall ist für den Fluß Tjiliwoeng kommen hier keine Fabriken vor, die ihren Abfall in den Fluss entleeren, so daß diese Ursache der großen Armut an Planktonorganismen von verschiedenen Strömen in Europa hier nicht besteht.

Wie alle Ströme auf Java dient auch dieser zum Defäzieren, Waschen, Baden usw., so daß wir hier dasselbe Bild haben, welches wir früher 
rom Tjiliwoengfluß gegeben haben. Nur kommt hier kein Laterit vor, dalier ist die Farbe also dunkelgrau. In einem Reagenzglas ist das Wasser trübe, und schon auf $100 \mathrm{ccm}$ kann man nach 24 Stunden einen Niederschlag ron Detritus und Schlick sehen.

Das Wasser kommt größtenteils von den Sawahs ${ }^{1}$ ). Wir wissen, daß das Wasser der Sawahs im allgemeinen vornehmlich Desmidiaceen enthält, weiter stammt ein Teil des Wassers der Tjimoeloe ron den Bergströmen, woron wir jetzt auch einigermaßen die qualitative Zusammenstellung kennen.

Die Ergebnisse für die Tjimoeloe sind folgende:

3. November 1920. Pro $100 \mathrm{I}$ Wasser $21 / 2$ ccm Satz.

Beinahe nur Schlick, sehr wenig Detritus.

Nitzschia sigmoidea (Nitzsch) W. Smith.

Cathypna luna Ehrenberg.

18. Norember 1920. Pro 1001 Wasser $2 \mathrm{ccm}$ Satz.

Viel Scblick, wenig Detritus.

Surirella splendida Kuetzing.

Naricula limosa Kuetzing.

" ambigua (Ebrenberg).

Melosira granulata (Ehrenberg) Ralfs var. angustissima

o. Mueller.

7. Dezember 1920. Pro 1001 Wasser $2 \frac{1}{2}$ ccm Satz.

Beinahe ausschließlich Schlick und Detritus.

Naricula rhycocephala Kuetzing.

Trachelomonas hispida (Perty) Stein.

Surirella splendida (Kuetzing.

21. Dezember 1920. Pro $100 \mathrm{l}$ Wasser $1 \mathrm{ccm}$ Satz.

Viel Schlick und Detritus.

Surirella splendida Kuetzing.

Nitzschia linearis (C. A. Agardb) W. Smith.

Bacillaria paradoxa (Gmelin) Gruenow.

Naricula radiosa Kuetzing.

6. Januar 1921. Pro $100 \mathrm{I}$ Wasser $31 / 2 \mathrm{ccm}$ Satz.

Viel Schlick und Detritus.

Surirella splendida Kuetzing.

Difflugia lobostoma Leidy.

1) Sawahs $=$ Reisfelder; vgl. van Oye, Int. Rev. X, S. 14. 
15. Januar 1921. Pro 1001 Wasser $2 \mathrm{ccm}$ Satz.

Viel Schlick, weniger Detritus.

Difflugia corona Wallich.

Surirella splendida Kuetzing.

Pleurosigma acuminatum (Kuetzing) Gruenow.

Gomphonema elegans Gruenow.

Phacus pleuronectes (O. F. Mueller) Dujardin.

Nitzschia sigmoidea (Nitzsch) W. Smith.

5. Februar 1921. Pro 1001 Wasser $2 \frac{1}{4} \mathrm{ccm}$ Satz.

Ausschließlich Schlick.

18. Februar 1921. Pro 1001 Wasser $1 \frac{1}{2} \mathrm{ccm}$ Satz.

Viel Schlick und Detritus.

Arcella vulgaris Ehrenberg.

Chrooccocus indicus Zeller.

"

turgidus (Kuetzing) Naegeli.

28. Februar 1921. Pro 1001 Wasser $3 / 4$ com Satz.

Viel Schlick, weniger Detritus.

Oscillatoria major Vaucher.

Arcella vulgaris Ehrenberg.

Surirella splendida Kuetzing.

Aphanothece minuta Wallroth.

Phacus pyrum (Ehrenberg) Stein.

Navicula viridis (Nitzsch) Kuetzing.

10. März 1921. Pro 1001 Wasser $1 \mathrm{ccm}$ Satz.

AusschlieBlich Schlick und Detritus.

30. März 1921. Pro $100 \mathrm{I}$ Wasser $1 \frac{1}{2}$ ccm Satz.

Beinahe ausschließlich Detritus und auch viel Schlick.

Vanheurckia rhomboides Ehrenberg.

23. April 1921. Pro 1001 Wasser 2 com Satz.

Ausschließlich Schlick und Detritus.

4. Mai 1921. Pro $100 \mathrm{l}$ Wasser 1\% ccm Satz.

Sehr viel Schlick und Detritus.

Surirella splendida Kuetzing.

10. Mai 1921. Pro 1001 Wasser $2 \frac{1}{4}$ cem Satz.

Viel Schliek und Detritus. 
Closterium acerosum Ehrenberg.

Surirella ovalis Brébisson var. ovata Kuetzing.

Surirella spleadida Kuetzing.

Encyonema caespitosum Kuetzing.

Spirogyra in degeneriertem Zustand.

30. Mai 1921. Pro $100 \mathrm{l}$ Wasser $1 \frac{1}{2}$ cem Satz.

Schlick und Detritus.

Melosira granulata (Ehrenberg) Ralfs var. angustissin

o. Mueller.

Nitzschia microcephale Gruenow.

Mastogloia grevillei W. Șmith.

Viel Diatomeen.

Cladocera ein Exemplar.

7. Juni 1921. Pro 1001 Wasser 2 cem Satz.

Wenig Schlick und Detritus.

Conferoa spec. in degeneriertem Zustand.

Difflugia corona Wallich.

Difflugia lobostoma Leidy.

Oscillatoria princeps Vaucher.

Melosira varians C. A. Agardh.

Epithemia ocellata Kuetzing.

Synedra ulna (Nitzsch) Ehrenberg.

20. Juni 1921. Pro $100 \mathrm{I}$ Wasser $2 \frac{1}{2} \mathrm{ccm}$ Satz.

Viel Schlick und Detritus.

Melosira granulata (Ehrenberg) Ralfs.

Surirella ovalis Brébisson var. ovata Kuetzing.

Schizostauron treubii Gutwinski.

Qualitativ haben wir also:

Myxophyceae.

Chrooccocus indicus Zeller.

$" \quad$ turgidus (Kuetzing) Naegeli.

Oscillatoria major Vaucher.

" princeps Vaucher.

Aphanothece minuta Wallroth.

Diatomeae

Surirella splendida Kuetzing.

" ovalis Brobisson var. orata Kuetzing. 
Navicula limosa Kuetzing.

" ambigua Ehrenberg.

$"$ radiosa Kuetzing.

" viridis (Nitzsch) Kuetzing.

Melosira granulata (Ehrenberg) Ralfs.

$"$ var. angustissima 0 . Mueller.

" varians C. A. Agardh.

Navicula rhyncocephala Kuetzing.

Nitzschia linearis (C. A. Agardh) W. Smith.

" microcephala Gruenow.

" sigmoïdea (Nitzsch) W. Smith.

Bacillaria paradoxa (Gmelin) Gruenow.

Gomphonema elegans Gruenow.

Epithemia ocellata Kuetzing.

Pleurosigma acuminatum (Kuetzing) Gruenow.

Synedra ulna (Nitzsch) Ehrenberg.

Vanheurckia rhomboïdes Ehrenberg.

Fncyonema caespitosum Kuetzing.

Mastogloia grevillei W. Smith.

Schizostauron treubii Gutwinski.

Desmidiaceae.

Closterium acerosum Ehrenberg.

Chlorophyceae.

Spirogyra spec. in degeneriertem Zustand.

Conferva spec. id.

(Keine lebendige Exemplare)

Rhizopoda.

Arcella rulgaris Ehrenberg.

Difflugia lobostoma Leidy.

$"$ corona Wallich.

Flagellata.

Trachelomonas hispida (Perty) Stein.

Phacus pleuronectes (O. F. Mueller) Dujardin.

$"$ pyrum (Ehrenberg) Stein.

Copepoda.

Keine.

Cladocera.

Ein Exemplar. 


$$
\text { Rotatoria. }
$$

Cathypua luna Ehrenberg.

Bis jetzt wurden also 38 Mikroorganismen im Plankton der Tjimoeloe bei Tasikmalaja gefunden, und zwar:

$$
\begin{aligned}
& \text { Myхophyceae . . . } 5 \\
& \text { Diatomeae . . . } 22 \\
& \text { Desmidiaceae. . . . } 1 \\
& \text { Chlorophy̌ceae . . . (2) } \\
& \text { Rizopoda . . . . } 3 \\
& \text { Flagellata . . . . } 3 \\
& \text { Cladocera . . . . } 1 \\
& \text { Rotatoria . . . . } 1
\end{aligned}
$$

Aus den Ergebnissen ist zu ersehen, daß vor allen anderen Gruppen an erster Stelle die Diatomeen in der Tjimoeloe vorkommen, während die Chlorophyceen ganz und gar verschwinden und von den Desmidiaceen nur eine einzige Art gefunden wurde, dagegen kommen die Myxophyceen hier häutiger vor, und ron den Flagellaten werden auch verschiedene Arten angetroffen. Cupepoden kommen nicht mehr vor, während Rotatorien äußerst selten sind und ron den Cladoceren nur ein Exemplar gefunden wurde. Also auf ungefähr $15 \mathrm{~km}$ Entfermung haben wir hier ein ganz anderes Bild als vom Bergstrom Tjikoenir.

Die qualitativen Änderungen sind sehr begreiflich. Tn diesem Miliea, w1) nur diffuses Licht, viel organische Stoffe und Abfall rorkommen und das arm an Sauerstoff ist, können die Chlorophyceen nicht weiterleben und gehen zugrunde. Die Desmidiaceen hingegen berorzugen stillstehendes Wasser, während für die Copepoden und die Rotatorien weder Boden- noch Ufervegetation vorhanden ist. Im Tjikoenir dagegen kommen viele steine mit Polstern von Chlorophyceen vor, zwischen welchen allerlei Insektenlarven, Rundwürmer, Rotatorien, Copepoden usw. zu finden sind.

$\mathrm{DaB}$ sich in einem an organischen Stoffen so reichem Bezirke wie dem Wasser der Tjimoeloe auch Arten der mehr polysaproben Gruppen, nämlich Myxophyceen und Flagellaten entwickeln, kann ebenfalls kein Erstaunen erregen.

Dies alles wäre sehr begreiflich, wenn nicht neben diesen Ergebnissen auch noch unsere Befunde des Tjiliwoeng-Flusses vorhanden wären. Doch glaube ich, daß bei näherer Betrachtung alle Tatsachen sehr begreiflich sind, wenn man die ökologischen Umstände tiefer ergründen will. 
Daß ich damals die Ergebnisse des Tjiliwoeng-Flusses ${ }^{1}$ ) nicht erklären konnte, lag daran, daß ich die Verhältnisse der übrigen Gewässer Javas noch nicht kannte, so daß ein Vergleich nicht möglich war.

In den untiefen Flachlandströmen sind die Lebensverhältnisse so ungünstig, daß fast keine Mikroorganismen sich entwickeln können oder am Leben bleiben; nur die Diatomeen, die mit dem Ursprungswasser gekommen sind, bleiben übrig, während Polysaprobien sich entwickeln.

In den tiefen Flachlandströmen sind die ökologisehen Verbältnisse etwas anders. Die Wassermenge ist viel größer, und als Folge ist die Verunreinigung weniger tiefgreifend, obwohl quantitativ viel mehr Abfall im Strom kommt. Die Dilution der gelösten organischen Stoffe und des Abfalls im allgemeinen ist eine sehr grofe; die Folge ist, daß unter gewissen Umständen, besonders in den Monaten, in welchen im Ursprungswasser einige Arten in sehr großer Anzabl vorkommen, diese in den tiefen Flachlandströmen noch am Leben bleiben können, während sie in den untiefen Flachlandströmen zugrunde gehen. Arten wie die Ceratium hirundinella (0. Mueller) Schrank, die wir im Tjiliwoeng-Fluß während des Monats März antrafen, sind also keine antochthone Planktonten der Flüsse auf Java, aber können, wenn sie ron ihrem ursprünglichen Standort in einen tiefen Flachlandstrom kommen, ohne erst einen untiefen Flachlandstrom durchgehen zu müssen, am Leben bleiben.

Die eine Desmidiacee, die wir in der Tjimoeloe fanden, darf uns auch nicht irreleiten, sie stammt aus einem Monat, wo in den verschiedenen Sitoes ${ }^{2}$ ) der Umgegend von Tasikmalaja die Chlorophyceen und speziell die Desmidiaceen vorwiegend gefunden wurden.

Um eine Vorstellung von der Fülle von Desmidiaceen zu geben, die in dieser Zeit in der Sitoe Tjibeureum Gobras vorkamen, lasse ich hier die Liste der am 8. April 1921 in dieser Sitoe gefundenen Arten folgen: Arthrodesmus convergens Ehrenberg.

Cosmarium tinctum Ralfs.

pyramidatum Brébisson.

Xanthidium antilopaeum Kuetzing f. javanicum Nordstedt. Staurastrum dejectum Brébisson.

Micrasterias foliacea Bailey.

" $\quad$ max melitensis Hassall.

1) $\operatorname{ran}$ Oyc, P., Int. Rev. IX, S. 160 .

2) vgl. Int. Rev. X, S. 16. 
Gonatozygon pilosum Wolle.

Hyalotheca dissiliens Brébisson.

Closterium kuetzingii Brébisson.

Staurastrum proboscideum (Brébisson) Archer.

Mougeotia genuflexa (Dillwyn) Agardh var. gracilis (Kuetzing)

Reinsch.

Staurastrum sexangulare (Bulnheim) Rabenhorst.

Das ganze Material war fast wie eine Reinkultur ron Desmidiaceen. Ferner muß ich auf Grund meiner anderen Beobachtungen annehmen, dab das Plankton der Reisfelder der Umgegend zu dieser Zeit auch vorwiegend aus Desmidiaceen bestand.

Aus oben angefïhrten Tatsachen können wir mit Sicherheit schließen, daß die einzige im Monat Mai gefundene Desmidiacee zufälligerweise im Plankton der Tjimoeloe rorkam. Sie soll uns also im Ziehen der Schłüsse nicht irreführen. Man wundert sich im Gegenteil, daß nicht mehrere Desmidiaceen, wenn auch in degeneriertem Zustand, in der Tjimoeloe gefunden wurden.

Was hier ron den Desmidiaceen gesagt ist, gilt auch für die übrigen Chloropbyceen, wovon nur zwei Exemplare und dann noch in degene. riertem Zustand gefunden wurden.

Daß die oben angegebenen ökologischen Faktoren anch wirklich die qualitative Zusammenstellung des Potamoplanktons der untiefen Flachlandströme auf Jara bedingen, geht noch aus folgenden Beobachtungen herror.

Die Flüsse Javas zeigen in ihrem Lauf auf rerschiedenen Stellen sugenannte Leuwis; dies sind tiefere Plätze, wo das Wasser riel langsamer strömt. Zur Zeit kann ich über das Plankton ron diesen Leuwis und seinem Einfluß auf das Potamoplankton noch nichts sagen, denn die Leuwis selbst sind zu rerschieden, und ich babe noch nicht gen ügende Beobacbtungen, um eine Folgerung zu ermöglichen. Jedoch kann ich hier schon mitteilen, daß das Plankton der Leuwis ganz anders ist als das gewöhnliche Potamoplankton.

So fand ich am 10. Juni 1921, drei Tage nachdem ich zum ersten Male eine Desmidiacee in der Tjimoeloe gefunden batte, folgende Arten in einer Leuwi des Stroms Tjiboganti bei Gampelang, ungefähr $12 \mathrm{~km}$ ron Tasikmalaja entfernt. Auf $100 \mathrm{l}$ Wasser kam nur ein setzrolumen von $1 / 4 \mathrm{ccm}$. Dieses war fast eine Reinkultur ron Pediastrumarten, gemischt mit einigen anderen Chlorophyceen und einigen Flagellaten. 
Die Liste der gefundenen Planktonten ist:

Pediastrum boryanum (Turpin) Meneghini.

$" \quad " \quad " \quad$ " $\quad " \quad$ f. integrum Naegeli.

Pediastrum duplex Meyen.

$" \quad " \quad \Rightarrow \quad$ var. asperum A. Braun.

Phacus pleuronectes (O. F. Mueller) Dujardin.

" longicaudatus (Ehrenberg) Dujardin.

Scenedesmus quadricauda (Turpin) Brébisson.

$" \quad$ perforatus Lemmermann.

Melosiragranulata(Ehrenberg) Ralfs var. angustissima O.Mueller. $" \quad$ varians C. A. Agardh.

Euglena deses Ebrenberg.

" oxyurus Schmarda.

Kirchneriella lunaris (Kirchner) Moebius.

Oocystis schroeteri Chodat.

Brachionus falcatus Zacharias.

Trachelomonas schauinslandi Lemmermann.

Oscillatoria chlorina Kuetzing.

Copepoden einige.

Rotatorien einige.

Wie aus den angeführten Listen zu ersehen, ist die Zusammenstellung des Planktons der Leuwi, der Tjimoeloe und des Flusses Tjikoenir im selben Monat zu verschieden voneinander, um hier nicht die obengenannten Faktoren als maßgebend anzuerkennen.

Erwähnt soll noch werden, daß kein Leuwi in der Tjimoeloe vorkommt auf einer Entfernung von mehr als $10 \mathrm{~km}$ stromaufwärts vom Platze, wo die Proben stets genommen wurden.

Während also in den Sitoes und Leuwis der Umgegend von Pasikmalaja viel Chlorophyceen und Desmidiaceen vorkamen, fanden wir in dem untiefen Flachlandstrom Tjimoeloe keine einzige Chlorophycee und nur eine Desmidiacee. Diese Tatsachen sind so auffallend, daß man sie beinahe als theoretische Beispiele annehmen könnte.

Die quantitativen Ergebnisse der Setzvolumina der Tjimoeloe sind: auf 1001 Wasser

3. Norember $1920 \cdot 21 / 2 \mathrm{~cm}$

18. November .. . 2 "

7. Dezember . . $2 \frac{1}{2}$, 


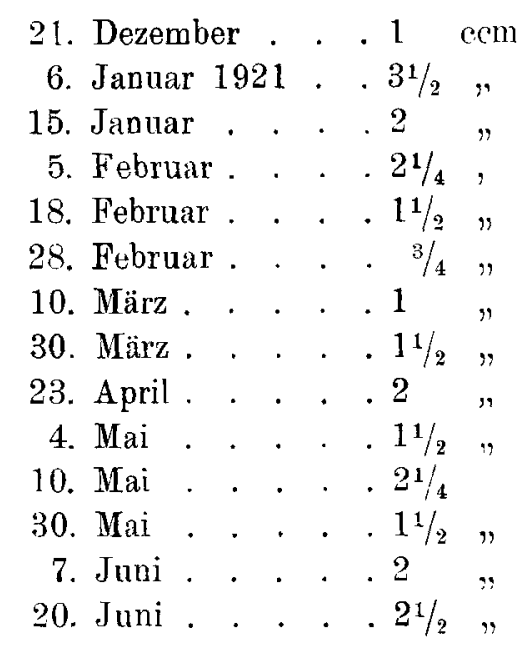

Wenden wir uns jetzt erst noch dem Bache zu. Dieser ist so unbedeutend, daß er keinen speziellen Namen hat; er läuft unweit von dem Flusse Tjikoenir, steht auch in direkter Verbindung mit diesem und ist kaum $1 \mathrm{~m}$ breit und $20-30 \mathrm{~cm}$ tief. Die Ränder sind mit Gras und Moos bewachsen, der Boden besteht aus gröberem Kies, das Wasser läuft ziemlich schnell.

Die Ergebnisse sind:

16. Oktober 1920. Pro $100 \mathrm{l}$ Wasser $1 / 2 \mathrm{ccm}$ Satz.

Viel Schlick, wenig Detritus.

Draparnaldia plumosa (Vaucher) C. A. Agardh.

Surirella splendida Kuetzing.

Cylindrocapsa involuta Reinseh.

Microspora amoena (Kuetzing) Rabenborst.

Arcella rulgaris Ehrenberg.

Stücke ron Moos.

17. November 1920. Pro 1001 Wasser $\% / 4 \mathrm{ccm}$ Satz.

Wenig Schlick und Detritus.

Anuraea aculeata Ehrenberg var. valga Ehrenberg.

Monostyla lunaris Ehrenberg.

Microspora stagnorum (Kuetzing) Lagerheim.

Surirella splendida Kuetzing.

Noteus quadricornis Ehrenberg.

Copepoden. 
8. Dezember 1920. Pro 1001 Wasser $1 \frac{1}{2}$. ccm Satz.

Viel Schlick und Detritus.

Spirogyra crassa Kuetzing.

Melosira granulata (Ehrenberg) Ralfs var. angustissima

o. Mueller.

Amphora ovalis Kuetzing.

Navicula ambigua Ehrenberg.

A rcella vulgaris Ehrenberg var. angulosa (Perty) Stein.

Trachelomonas Westi Jadwiga Woloszynska.

Mastogloia smithii Thwaites.

Centritractus belanophora (Schmidle) Lemmermann.

Surirella splendida Kuetzing.

Synedra ulna (Nitzsch) Ehrenberg var. splendens Kuetzing.

7. Januar 1921. Pro $100 \mathrm{I}$ Wasser $13 / 4$ ccm Satz.

Viel Schlick und Detritus.

Pleurosigma acuminatum Gruenow.

Difflugia lanceolata Pénard.

Surirella splendida Kuetzing.

Cladophora glomerata (Linnaeus) Kuetzing.

11. Februar 1921. Pro $100 \mathrm{l}$ Wasser $2 \mathrm{ccm}$ Satz.

Schlick und Detritus.

Surirella splendida Kuetzing.

Pleurosigma spencerii (J. Quekett) W. Smith.

Navicula brebissonii Kuetzing.

Mastogloia smithii Thaites.

Epithemia sorex Kuetzing.

Melosira varians C. A. Agardh.

2. April 1921. Pro 1001 Wasser $13 / 4$ cem Satz.

Schlick und Detritus.

Asplanchna priodonta Gosse.

Anuraea aculeata Ehrenberg var. valga Ehrenberg.

Synchaeta tremula Ehrenberg.

24. Mai 1921. Pro $100 \mathrm{l}$ Wasser $1 / 4 \mathrm{ccm}$ Satz.

Seblick und Detritus.

Spirogyra spec.

Conferva affinis Kuetzing. 
9. Juni 1921. Pro $100 \mathrm{l}$ Wasser $1 \mathrm{ccm}$ Satz.

Wenig Schlick und Detritus.

Surirella splendida Kuetzing.

Arcella vulgaris Ehrenberg.

Spirogyra.weberi Petit.

Bacillaria paradoxa (Gmelin) Gruenow.

Melosira granulata (Ehrenberg) Ralfs.

Difflugia lobostoma Leidy.

20. Juni 1921. Pro 1001 Wasser $1 / 2$ cem Satz.

Sehr wenig Schlick und Detritus.

Melosira varians C. A. Agardh.

Anuraea aculeata Ehrenberg var. valga Ehrenberg.

Triarthra terminalis Plate.

Melosira granulata (Ehrenberg) Ralfs var. angustissima

o. Mueller.

Phacus longicaudatus (Ehrenberg) Dujardin.

Scenedesmus quadricaud (T'urpin) Brébisson.

Cosmarium subcrenatum Hantzsch.

Pediastrum duplex Meyen.

Brachionus quadratus Rousselet.

Polyarthra platyptera Ehrenberg.

Qualitativ sehen wir also:

$$
\begin{gathered}
\text { Myxophyceae: } \\
\text { Keipe. } \\
\text { Diatomeae. }
\end{gathered}
$$

Surirella splendida Kuetzing.

Melosiragranulata (Ehrenberg) Ralfs var. angustissima O.Mueller.

" granulata (Ehrenberg) Ralfs.

Amphora ovalis Kuetzing.

Navicula ambigua Ehrenberg.

" brebissonii Kuetzing.

Mastogloia smithii Thaites.

Synedra ulna (Nitzsch) Ehrenberg var. splendens Kuetzing.

Pleurosigma acuminatum Gruenow.

" spencerii (J. Quekett) W. Smith.

Epithemia sorex Kuetzing.

Melosira varians C. A. Agardh.

Bacillaria paradoxa (Gmelin) Gruenow. 
Desmidiaceae.

Cosmarium suberenatum Hantzsch.

Chlorophyceae.

Draparnaldia plumosa (Vaucher) C. A. Agardh.

Microspora amœna (Kuetzing) Rabenhorst.

" stagnorum (Kuetzing) Lagerheim.

Spirogyra crassa Kuetzing.

" weberi Petit.

Cladophora glomerata (Linnaeus) Kuetzing.

Conferva affinis Kuetzing.

Scenedesmụs quadricauda (Turpin) Brébisson.

Pediastrum duplex Meyen.

Cylindrocapsa involuta Reinsch.

Centritractus belanophora (Schmidle) Lemmermann.

Flagellata.

Trachelomonas Westi Jadwiga Woloszynska.

Phacus longicaudatus (Ehrenberg) Dujardin.

Rhizopoda.

Arcella vulgaris Ehrenberg.

Difflugia lanceolata Pénard.

var. angulosa (Perty) Stein.

$"$ lobostoma Leidy.

Copepoda.

?

Rotatoria.

Anuraea aculeata Ehrenberg var. valga Ehrenberg.

Monostyla lunaris Ehrenberg.

Noteus quadricornis Ehrenberg.

Asplanchna priodonta Gosse.

Synchaeta tremula Ehrenberg.

Triarthra terminalis Plate.

Brachionus quadratus Rousselet.

Polyarthra platyptera Fhrenberg.

Es wurden also bis jetzt in dem Bache 39 Mikroorganismen im Plankton gefunden, und zwar:

Myxophyceae . . 0

Diatomeae . . . 13

Desmidiaceae . . . 1

Chlorophyceae . . . 11
Flagellata . . . . 2

Rhizopoda . . . 4

Copepoda . . . . . ?

Rotatoria . . . . 8 
In großen Zügen ist die qualitative Zusammenstellung des Planktons dieses Baches dieselbe wie in dem Strom Tjikoenir. Es kommen jedoch die Rhizopoden etwas weniger häufig vor, während von den Rotatorien mehrere Arten und in größerer Anzahl rorhanden sind. Die Chlorophyeeen hingegen sind nicht so zahlreich. Dies stimmt mit unseren rorhergehenden Folgerungen. Das Wasser ist noch nicht mit Schlamm und Detritus derartig verunreinigt, daß die Chloropbyceen wie Draparnaldia, Microspora, Cladophora und andere hier nicht mehr am Leben bleiben könnten, doch das Wasser fließt viel langsamer, s' daB die Rotatorien sich am Ufer besser entwickeln können und so ins Plankton des fließenden Wassers gelangen.

Die Ufer sind, wie schon erwähnt, mit Moos und Gras bewachsen, worin wir die gewöhnliche Mlikroflora und fauna der Moosrasen antreffen, die gelegentlich im Wasser des Baches vorkommen kann.

Die kleine Anzahl Rhizopodenarten erregt einigermaßen Erstaunen. Ich kann hier nur mitteilen, daß die Rhizopoden numerisch zahireicher sind als im Fluß Tjikoenir, doch das schlieBt nicht aus, daß man auch eine größere Anzahl ron Arten erwarten könnte.

Desmidiaceen kommen praktisch gesprochen nicht ror, die eine gefundene Art kann nicht anders als zufällig ins Wasser gekommen sein. Dies ist auch sehr natürlich. denn woher würden die Desmidiaceen kommen? Das Wasser stammt aus einem brausenden Bergstrom, wie wir gesehen haben, und Sawahwasser hat den Bach noch nicht verunreinigt.

Also haben wir eine wenig größere Anzahl Diatomeen, numerisch etwas mebr Rbizopoden, der Artenzahl nach aber etwas weniger, mebr Rotatorien und etwas weniger Chlorophyceen.

Mit anderen Worten, eine qualitative Zusammenstellung des Planktons, welche zwischen dem Teichplankton und dem brausenden Bergstromplankton steht.

$\mathrm{Da}$ uun, wie leicht begreiflich ist, die ökologischen Verhältnisse in dem Bache auch als eine Mischung von beiden oben erwähnten Bezirken anzusehen sind, müssen wir annelmen, daß unsere Folgerungen betreffs des Einflusses der in Frage kommenden Faktoren auch richtig sind. Das eine ist eine logische Folge vom anderen. Aus allen Ergebnissen ersehen wir auch, daß der schnelffließende Bach noch zu dem brausenden Bergstrome gerechnet wẹrden mußB.

Die quantitativen Ergebnisse der Setzrolumina auf 1001 Wasser sind folgende: 
16. Oktober 1920 . $1 / 2 \mathrm{ccm}$

17. November . . $3 / 4$,

8. Dezember . . $11 / 2$,

7. Januar $1921 \cdot 13 / 4 "$

-11. Februar . . 2 ,
2. April . . . $13 / 4 \mathrm{ccm}$

24. Mai . . . 1/4 "

9. Juni . . . 1 "

20. Juni . . . 1/2

Bevor an die Vergleichung und Besprechung aller Ergebnisse herangegangen wird, wollen wir erst den mittleren Wert des Regenfalls in Tasikmalaja anführen, weiter auch den Regenfall während der Monate Oktober 1920 bis Juni 1921 und endlich den täglichen Regenfall wäbrend dieser Monate. Der Regenfall ist ja, wie ich schon offters gezeigt habe, der wichtigste Faktor in der Biologie des tropischen Planktons.

Der monatliche mittlere Wert des Regenfalles in Tasikmalaja, berechnet aus 29 Jahren, ist in $\mathrm{mm}^{1}$ ):

I. II. III. IV. V. VI. VII. VIII. XI. X. XI. XII. $\begin{array}{llllllllllll}366 & 383 & 493 & 356 & 299 & 233 & 196 & 177 & 206 & 368 & 392 & 398\end{array}$

Diese Ziffern geben folgende Kurve:

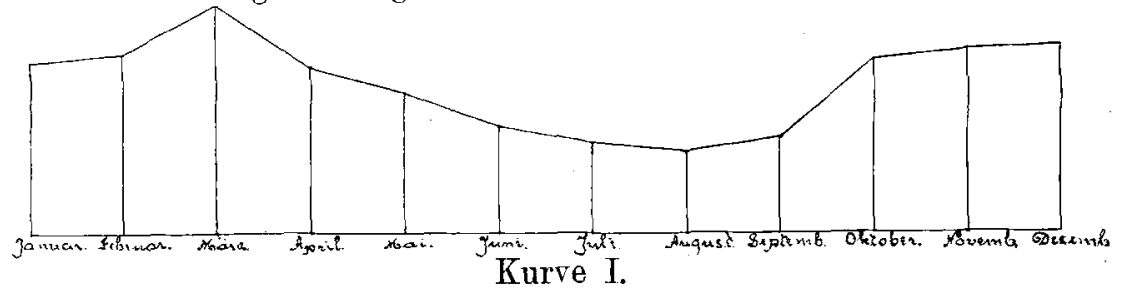

Die Werte für den Regenfall während der Monate Oktober 1920 bis Juni 1921 sind folgende:

VIII. IX. X. XI. XII. I. II. III. IV. V. VI. $\begin{array}{lllllllllll}316 & 397 & 354 & 248 & 299 & 306 & 237 & 587 & 141 & 70 & 153\end{array}$

Diese Ziffern geben folgende Kurve:

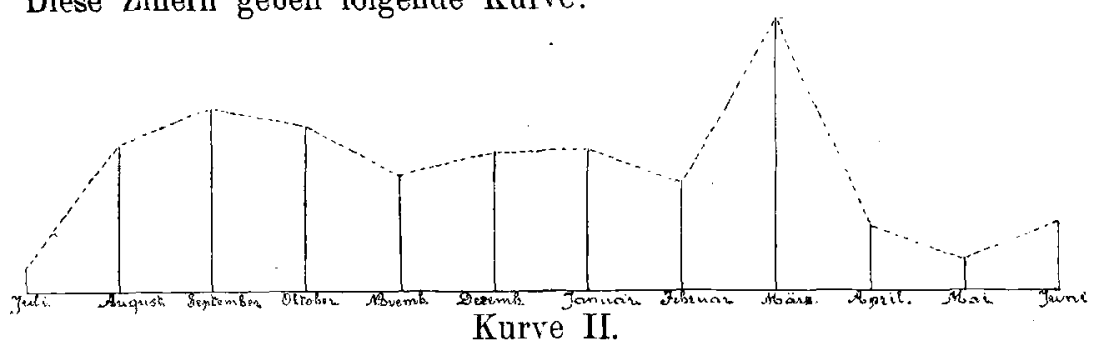

Der Vergleich der zwei Kurven lehrt uns, daß der Regenfall während der betreffenden Monate ziemlich stark vom normalen Regenfall abwich, was uns noch deutlicher in folgender graphischen Darstellung wird:

1) Regenwarnemingen in Nederlandsch. Indië bewerkt en nitgegeven door het konink. magnetisch en meteorologiseh Observatorium te Batavia. Jaarg. 37. 1915. s. 154 . 




Kurve 111.

Endlich sind die Ziffern des täglichen Regenfalls in Tasikmalaja während den Monaten September 1920 bis Juni 1921 folgende:

\begin{tabular}{|c|c|c|c|c|c|c|c|c|c|c|}
\hline & IX. & $\mathrm{X}$ & XI. & XII. & I. & II. & III. & IV. & V. & VI. \\
\hline 1 & 30 & - & - & - & 9 & 39 & 3 & 5 & - & - \\
\hline 2 & - & - & - & - & - & 9 & 65 & - & - & 1 \\
\hline 3 & - & - & - & 5 & 2 & - & 39 & 35 & - & 8 \\
\hline 4 & 13 & - & - & 15 & 32 & - & 7 & 4 & - & 46 \\
\hline 5 & 4 & - & - & 8 & 20 & 41 & - & - & - & 10 \\
\hline 6 & 21 & - & - & 30 & 29 & - & - & - & 7 & 13 \\
\hline 7 & 10 & - & 21 & 15 & 23 & 34 & 22 & - & - & - \\
\hline 8 & - & - & 6 & 23 & 24 & 5 & 3 & - & - & - \\
\hline 9 & 5 & - & - & 9 & 3 & 2 & - & 10 & 1 & - \\
\hline 10 & - & - & 54 & 72 & - & 16 & 7 & - & - & - \\
\hline 11 & 63 & - & - & 23 & - & 5 & 57 & 1 & 3 & - \\
\hline 12 & 16 & - & - & 2 & - & 29 & 10 & 1 & - & - \\
\hline 13 & 6 & - & - & 2 & 2 & 5 & 57 & 4 & - & - \\
\hline 14 & 11 & - & 3 & - & 16 & 37 & 12 & 4 & - & - \\
\hline 15 & - & - & 6 & - & 11 & - & 16 & - & $\ldots$ & - \\
\hline 16 & $\ldots$ & 37 & 10 & - & 17 & $\ldots$ & 3 & - & - & 13 \\
\hline 17 & 3 & 63 & - & 3 & - & - & - & - & - & - \\
\hline 18 & - & 23 & 6 & 8 & 2 & - & -- & 4 & - & - \\
\hline 19 & - & 17 & 3 & - & - & - & 14 & 5 & - & - \\
\hline 20 & 3 & 2 & 40 & - & - & - & 43 & - & - & - \\
\hline 21 & - & 5 & 50 & - & - & - & 6 & - & 5 & 4 \\
\hline 22 & 32 & 30 & - & - & - & 5 & - & 12 & 17 & 2 \\
\hline 23 & 48 & 90 & - & 4 & - & -- & 27 & - & - & 18 \\
\hline 24 & - & 40. & 7 & 5 & - & - & 35 & - & - & - \\
\hline 25 & - & 11 & 14 & 5 & 12 & - & 9 & - & - & - \\
\hline 26 & - & 3 & 19 & 20 & 49 & 6 & 12 & - & 36 & - \\
\hline 27 & 40 & 19 & - & 3 & - & - & 43 & - & - & - \\
\hline 28 & 40 & 13 & 6 & 34 & - & 4 & 18 & 5 & - & 17 \\
\hline 29 & 40 & 1 & 3 & -. & - & - & 49 & 14 & - & 21 \\
\hline 30 & 12 & - & - & - & 3 & - & 30 & - & - & - \\
\hline 31 & - & - & - & 4 & 42 & - & - & - & 1 & - \\
\hline
\end{tabular}


Bringen wir nun zum leichteren Vergleich alle unsere Ergebnisse in Tabellen, dann bekommen wir:

\begin{tabular}{|c|c|c|c|c|c|c|c|c|c|c|}
\hline & & $\mathrm{X}$. & XI. & XII. & I. & II. & III. & IV. & V. & VI. \\
\hline \multirow{6}{*}{ Tjikoenir } & Myxophyceae & - & - & 1 & 一 & - & 一 & - & - & - \\
\hline & Diatomeae & 3 & 1 & 3 & 5 & 2 & - & 2 & 1 & 4 \\
\hline & Desmidiaceae . & - & - & - & - & - & - & - & - & 1 \\
\hline & Chlorophyceae. & 5 & 3 & 2 & 4 & - & 一 & - & 2 & 7 \\
\hline & Rhizopoda . . . & - & - & - & 2 & 2 & - & 1 & - & 5 \\
\hline & Rotatoria.... & - & 2 & - & - & - & - & 1 & - & - \\
\hline \multirow{7}{*}{ Tjimoeloe } & Myxophyceae. & - & - & - & - & 4 & - & - & - & 1 \\
\hline & Diatomeae ... & - & 5 & 5 & 4 & 2 & 1 & 1 & 6 & 6 \\
\hline & Desmidiaceae . & -- & - & - & - & - & - & - & 1 & - \\
\hline & Chlorophyceae. & - & 一 & - & 一 & - & - & - & 1 & 1 \\
\hline & Flagellata ... & - & - & 1 & 1 & 1 & - & - & - & - \\
\hline & Rhizopoda . . . & - & 一 & - & 2 & 1 & - & - & - & 2 \\
\hline & Rotatoria... & - & 1 & 一 & - & - & 一 & - & 一 & - \\
\hline \multirow{17}{*}{ Bach. } & Myxophyceae & 1 & $\ldots$ & - & - & - & - & - & - & 一 \\
\hline & Diatomeae ... & 1 & 1 & 6 & 2 & 6 & - & - & 一 & 5 \\
\hline & Desmidiaceae . & - & $\ldots$ & 一 & $\ldots$ & - & - & - & - & 1 \\
\hline & Chlorophyceae. & 2 & 1 & 2 & 1 & - & 一 & - & - & 3 \\
\hline & Flagellata ... & 一 & - & 1 & 一 & - & 一 & - & 2 & 1 \\
\hline & Rhizopoda... & 1 & - & 1 & 1 & - & - & - & - & 2 \\
\hline & Rotatoria. . . & - & 3 & - & - & - & - & $\mathbf{3}$ & - & 4 \\
\hline & & & \multicolumn{2}{|c|}{ Tjikoenir } & Tjimoeloe & \multicolumn{2}{|r|}{ Bach } & & & \\
\hline & \multicolumn{2}{|c|}{ Myxophyceae } & \multicolumn{2}{|c|}{1} & 5 & & 0 & & & \\
\hline & \multirow{2}{*}{\multicolumn{2}{|c|}{$\begin{array}{l}\text { Diatomeae . } \\
\text { Desmidiaceae }\end{array}$}} & \multicolumn{2}{|c|}{12} & 22 & & 13 & & & \\
\hline & & & \multicolumn{2}{|c|}{1} & 1 & & 1 & & & \\
\hline & & \multicolumn{2}{|c|}{12} & (2) & & 11 & & & \\
\hline & \multicolumn{2}{|c|}{ Flagellata .... } & \multicolumn{2}{|c|}{0} & 3 & & 2 & & & \\
\hline & \multicolumn{2}{|l|}{ Rhizopoda } & \multicolumn{2}{|c|}{6} & 3 & & 4 & & & \\
\hline & \multicolumn{2}{|l|}{ Copepoda } & \multicolumn{2}{|c|}{ - } & 0 & & ? & & & \\
\hline & \multirow{2}{*}{\multicolumn{2}{|c|}{ Cladocera }} & \multicolumn{2}{|c|}{ - } & 1 & & 0 & & & \\
\hline & & Rotatoria. & 3 & & 1 & & 8 & & & \\
\hline
\end{tabular}

Aus allen Beobachtungen ist zu ersehen, daß die Bergströme eine Periodizität aufweisen, die gerade wie die der Seen durch den Regenfall bedingt sein muß, denn sie kommt mit den Perioden von Regen und Trockenheit überein und zeigt auch Übergangsperioden.

Die qualitative Zusammenstellung ist jedoch eine ganz andere als diese der Sitoes, wie ich an anderer Stelle erörtern werde ${ }^{1}$ ).

1) van $\mathrm{Oy}$ e, P., Sur la périodicité du plancton d'eau douce à Java. In Vorbereitung. id. Oecologie du plancton des lacs à Java. Auch in Vorbereitung. 
Um die Biologie und Ökologie des Potamoplanktons gut zu begreifen, soll hier darauf hingewiesen werden, daß die Arten Draparnaldia, Stigeoclonium, Cladophora usw. äußerst selten und dann nur unter besonderen Umständen, also praktisch gesprochen gar nicht, in stillstehenden Gewässern vorkommen. Jedenfalls nie in Gewässern, die nicht ihr Wasser direkt von brausenden Bergströmen bekommen. Also kann man sagen, daß in typischen stillstehenden Gewässern, die nur durch Regenwasser gespeist werden, wie dies für den See von Pandjaloe der Fall ist, obengenannte Genera von Chlorophyceen nie vorkommen.

Dagegen sind in den stillstehenden Gewässern viel mehr Rhizopoden und Infusorien $z u$ finden, besonders zwischen Pflanzen wie Chara, Hydrilla usw.

Obwohl der Regenfall dieses Jabr unglücklicherweise sehr abnorm war, kann doch eine qualitative Periodizität der Bergströme beobachtet werden. Sie läßt sich zur Zeit folgendermaßen charakterisieren.

Numerisch kommen die Chlorophyceen am meisten vor in den Monaten Oktober, November und Dezember.

Die größte Anzahl von Diatomeen, speziell was die Indiriduen betrifft, findet man im Dezember, Januar und Februar, vermutlich auch im März.

Die Rotatorien kommen vornehmlich in den Monaten April und Mai vor.

Ob das Wiedervorkommen ron Chlorophyceen im Monat Juni eine zweite Chlorophyceenperiode einleitet oder der Anfang der im Dezember endigenden Periode ist, kann zur Zeit noch nicht gesagt werden.

Vergleichen wir nun diese Ergebnisse mit denen der Tjimoeloe und die des Tjiliwoengflusses ${ }^{1}$ ), dann sehen wir, daß die Bergströme ein eigenes Potamoplankton haben, das eine qualitative Periodizität aufweist, die abhängig ist von der Regen- und Trockenzeit. Weiter sehen wir, daß das Potamoplankton der Bergströme bis jetzt wenigstens drei Perioden in seiner qualitativen Erolution zeigt.

Das Plankton der Flachlandströme zeigt keine eigene Periodizität, es ist ganz und gar abhängig von dem Ursprungswasser.

Hierdurch erklärt sich auch, daß im März 1917 im Tjiliwoengflusse so viele Exemplare von Ceratium.hirundinella O. F. Mueller vorkamen. Dieselben entstammen den höher gelegenen Regionen, wo sie, wie unsere späteren Untersuchungen gelehrt haben, zu dieser Jahreszeit sebr häufig sind. Als mehr oder weniger charakteristisch für die Flachlandströme sind die Myxophyceen, die sich hier entwickeln.

1) van Oye, P., l. c. Int. Revue IX, 1920, S. 160. 
Sehr schematisch kann man also die Strŏme Javas folgendermaßen charakterisieren:

Bergströme vorwiegend katharobes Plankton neben einigen Oligosaproben.

Flachlandströme polysaprobes Plankton.

Man könnte auch noch sagen:

Bergströme: Chlorophyceenplankton,

Flachlandströme: Myxophyceen- und Flagellatenplankton, was allerdings nur eine sehr grobe Charakterisierung ist.

Bringen wir nun zum Vergleich die Ziffern der Setzyolumina in eine Tabelle, dann bekommen wir:

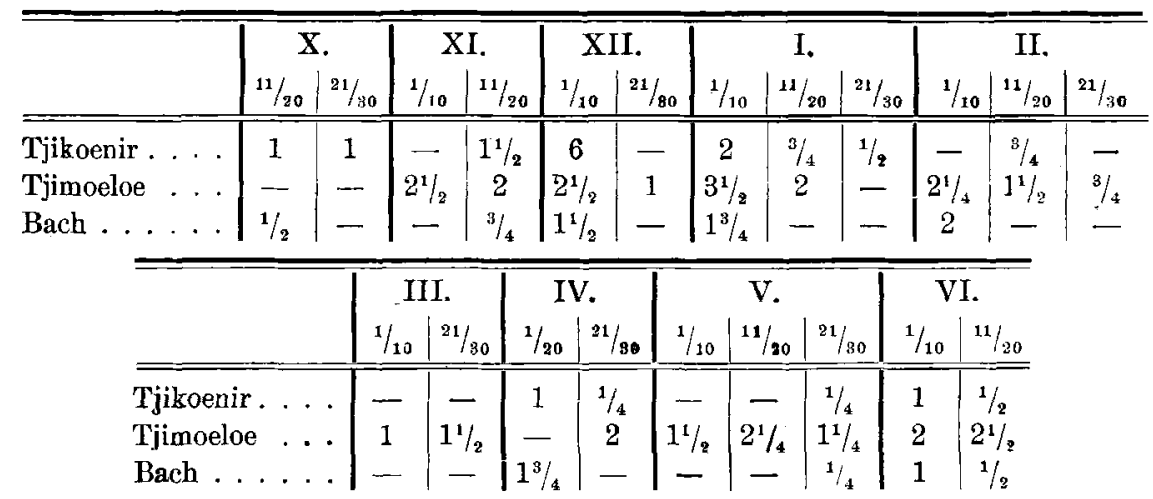

Wenn wir nun alle quantitativen Ergebnisse untereinander vergleichen, dann sehen wir: das Setzvolumen weder der Bergströme noch der Flachlandströme weist einige Abhängigkeit rom monatlichen Mittelwert des Regenfalles auf. Dies stimmt auch überein mit dem, was wir früher im Tjiliwoengflusse zu Batavia gefunden haben.

Alles wird jedoch deutlich, wenn wir den täglichen Regenfall der betreffenden Monate heranziehen; dann können wir feststellen, daß das Setzvolumen der Ströme, besonders der Bergströme, abhängig ist von dem täglichen Regenfall, und dies um so stärker, wenn der Strom ungestüm dahinfließt.

Wir haben hier also einen Gegensatz zu den Seen, in welchen das Plankton auch eine quantitative Evolution zeigt, die ganz und gar abhängig ist von der Kurve der mittleren monatlichen Werte des Regenfalles, wie ich später noch näher zөigen werde.

Hierbei ist auch zu erwähnen, daß die Periodizität der Seen eine Übereinstimmung in der qualitativen und quantitativen Evolution auf- 
weisen; während wir für die Ströme ja nur von einem Setzrolumen sprechen können, wobei der Schlamm den größten Teil einnimmt. Inwiefern aber die quantitative Entwicklung der einzelnen Arten numerisch in den Strömen eine Periodizität aufweist, kann zur Zeit noch nicht gesagt werden.

Außer der Biologie und Ökologie der strömenden Gewässer bringen uns die obenstehenden Ergebnisse auch eine nähere Einsicht in die Verhältnisse, unter welchen verschiedene Algen-Genera und -Familien in den Tropen vorkommen.

So hat sich Fritseh darüber gewundert, daß er so wenig Cladophora- und Rhizocloniumarten auf Ceylon gefunden hat. Zweimal fand er Cladophora und in beiden Fällen in brausendem (disturbed) Wasser, und ein drittes $\mathrm{Mal}$ in gut durchgelüftetem Wasser. Rhizoclonium dagegen fand er nur in: „estuaries and lagoons".

Soweit meine Untersuchungen von strömenden Gewässern auf Java, die, mit Ausnahme vom Tjiliwoengfluß, bis jetzt immer über $350 \mathrm{~m}$ stattfanden, werden die Beobachtungen von Fritsch über die Abwesenheit von Rhizoclonium und das Vorkommen von Cladophora, unter denselben Umständen, als er sie auf Ceylon fand, bestäligt.

Großes Erstaunen' muß jedoch der Satz dieses Autors erregen, wenn er sagt: "Draparnaldia has as yet only be recorded from the Sandwich Islands and Ecuador; it was not observed in Ceylon" $\left.{ }^{1}\right)$. Sicher können wir sagen, daß die Bemerkung von Fritsch unrichtig ist, wenn er behauptet: ,although there is nothing to show that these forms farour specially well aereted tropical waters". Wir fanden Draparnaldia ja sehr oft und üppig entwickelt in dem Strom Tjikoenir und gut lebende Exemplare in dem Bergbach.

Die wenigen Exemplare von Spirogyraarten, welche ich in den strömenden Gewässern Javas gefunden habe, besonders in Vergleich mit meinen übrigen Ergebnissen, machen es notwendig, das Vorkommen der zygnemaceae in den Gewässern Javas an anderem Orte zu besprechen; die Spirogyra sind ja Arten, die an erster Stelle in stillstehenden Gewässern vorkommen.

Dasselbe gilt für die Desmidiaceen, die sehr selten in strömenden Gewässern gefunden werden und also ebenfalls an einem anderen Platz besprochen werden sollen.

1) Fritsch, F. E., l. c. Ann. of Botan, S. 260. 
Zusammenfassend zeigen unsere Untersuchungen über die Biologie des Potamoplanktons auf Java folgende Ergebnisse:

1. Die Bergströme auf Java haben eine charakteristische Mikroflora.

2. Sie ist qualitativ die Folge von der reichlichen Anwesenbeit von Sauerstoff und der Helligkeit des Wassers.

3. Das Setzrolumen ist abhängig ron dem täglichen Regenfall, wobei vornehmlich Schlick das Volumen quantitativ beeinflußt.

4. Qualitativ kommt auch eine Periodizität vor, die folgende Stufen aufweist: Chlorophyceen, Diatomeen, Rotatorien.

5. Das Plankton besteht aus kataroben und oligosaproben Mikroorganismen.

6. Die Flachlandströme hingegen haben keine charakteristische Mikroflora:

7. Die qualitative Zusammensetzung ist teilweise abhängig von der des Ursprungswassers, insofern als nur jene Planktonten, die in eimem sauerstoffarmen und sehr trüben Wasser leben können, übrigbleiben.

8. Die Flachlandströme sind sehr arm an Mikroorganismen, da die meisten, welche den verschiedenen Ursprungsgewässern entstammen, in diesem Milieu mit wenig Licht und viel organischen Überresten nicht leben können. Folglich sind es meistens nur Diatomeen, Myxophyceen und Flagellaten, die hier angetroffen werden.

9. Hier kommen stark mesosaprobe und polysaprobe Mikroorganismen vor.

10. Das Setzvolumen ist ebenfalls, aber weniger ausgesprochen, rom täglichen Regenfall abhängig.

11. Eine qualitative Periodizität ist bis jetzt nicht mit Sicherbeit nachzuweisen.

12. Die Flachlandströme können gelegentlich Arten aufweisen, deren Vorkommen Erstaunen erregen könnte, doch bis jetzt ist ihre Anwesenheit noch immer erklärlich geworden, wenn das Ursprungswasser in Betracht gezogen wurde. Von einem eigenen Plankton außer den erwähnten Polysaproben ist hier keine Rede.

Tasikmalaja, Juli 1921. 